\title{
Worse Cardiovascular and Renal Outcome in Male SLE Patients: First Gender Study From the Swiss SLE Cohort Study
}

\section{Jelena Mihailovic}

Department of Nephrology and Hypertension Inselspital, University Hospital, Bern

\section{Camillo Ribi}

Division of Clinical Immunology and Allergy, University Hospital Lausanne, Lausanne

\section{Carlo Chizzolini}

Division of Clinical Immunology and Allergy, Department of Medicine, University Hospital and School of Medicine, Geneva

\section{Marten Trendelenburg}

Division of Internal Medicine and Clinical Immunology Laboratory, Department of Biomedicine, University Hospital Basel, Basel

\section{Johannes Von Kempis}

Division of Rheumatology and Immunology, Department of Internal Medicine, Kantonsspital St Gallen,

St Gallen

\section{Uyen Huynh-Do}

Department of Nephrology and Hypertension Inselspital, University Hospital, Bern

\section{Suzan Dahdal ( $\sim$ suzan.dahdal@insel.ch )}

Department of Nephrology and Hypertension Inselspital, University Hospital, Bern

\section{Research Article}

Keywords: SLE, Gender differences, disease manifestation, renal outcome, cardiovascular outcome

Posted Date: July 8th, 2021

DOI: https://doi.org/10.21203/rs.3.rs-645732/v1

License: (a) (1) This work is licensed under a Creative Commons Attribution 4.0 International License. Read Full License 


\section{Abstract}

Background: Systemic lupus erythematosus (SLE) in males is rare and poorly understood. Thus, still little is known about gender differences in SLE. We set out to identify gender differences regarding clinical manifestations as well as renal and cardiovascular outcomes of SLE.

Methods: We analyzed patient data from the Swiss SLE Cohort Study. Cumulative clinical manifestations according to the updated American College of Rheumatology criteria were recorded at inclusion.

Cardiovascular events were recorded within Systemic Lupus International Collaborating Clinics/American College of Rheumatology Damage Index (SLICC-SDI). Renal failure was defined as eGFR $<15$

$\mathrm{ml} / \mathrm{min} / 1.73 \mathrm{~m}^{2}$, initiation of renal replacement therapy or doubling of serum creatinine which were all assessed yearly or documented as end stage renal disease in SLICC-SDI. Risk differences were calculated using logistic regression and cox regression models.

Results: We analyzed 93 men and 529 women with a median follow up time of 2 years. Males were significantly older at diagnosis (44.4 versus 33.1 years, $p<0.001)$ and had less often arthritis $(57 \%$ versus $74 \%, p=0.001)$ and dermatological disorders (61\% versus $76 \%, p<0.01)$. In multivariate analysis female gender remained a significantly associated with arthritis and dermatological disorders. After adjusting for age, disease duration, ethnicity, time to diagnosis, medication and eGFR and SELENA SLEDAI at inclusion men had a significantly higher hazard ratio of 2.5 for renal failure (95\% confidence interval $(95 \%-\mathrm{Cl}) 1.1$ $6.0, p<0.04)$. Total SLICC-SDI Score was comparable. Men had significantly more coronary artery disease (CAD) $(17 \%$ versus $4 \%, p<0.001)$ and myocardial infarction ( $10 \%$ versus $2 \%, p<0.01)$. In multivariate analysis, male gender remained a significant risk factor for CAD (odds ratio (OR) 5.6, 95\%-Cl 2.3-13.7, $\mathrm{p}<0.001)$ and myocardial infarction (OR 8.3, 95\%-Cl 2.1-32.6, $\mathrm{p}=0.002)$.

Conclusion: This first gender study in a western European population demonstrates significant gender differences in SLE. Male gender is an independent risk factor for cardiovascular events and renal failure in SLE. Potential etiological pathomechanisms such as hormonal or X-chromosomal factors remain to be further investigated.

\section{Background}

Systemic lupus erythematosus (SLE) is a chronic autoimmune disease with a wide range of clinical manifestations and a potentially life-threatening outcome. As in other autoimmune diseases, women are much more affected than men are. An European study reported a female to male ratio of 10:1 [1]. As a consequence, male SLE is still poorly understood.

In the past decades, multiple studies showed striking evidence for gender differences which in turn aroused the interest in gender specialized medicine. Recent studies reported a higher mortality in males than females in systemic sclerosis, another autoimmune disease[2,3]. Thus, one could assume similar findings in SLE, and indeed, already in 1981 Wallace et al. reported a higher mortality in men with SLE than in women[4]. The more recent large LUMINA study showed a poorer long term prognosis with 
accelerated development of damage in men compared to women[5]. In the study of Roman et al. accelerated atherosclerosis was found to occur prematurely and independent of traditional risk factors for cardiovascular disease in SLE[6]. Cardiovascular disease is one of the main causes of death in SLE and indeed the LUMINA study identified male gender as a risk factor for cardiovascular events in patients with SLE[7]. Tan et al. showed in a large American cohort a higher rate of renal failure and end stage renal disease (ESRD) in males[8]. However, multiple other studies do not confirm these findings, especially regarding renal involvement and mortality[9]. For example, Renau et al. observed an increase in renal failure and death in females with SLE[10] and Voulgari et al. showed no overall increase in renal involvement in men[11]. Furthermore, ethnic background and socioeconomic status are known to influence the presentation and disease course of SLE[12]. The ethnic background of patients enrolled in the LUMINA study consists of only $28 \%$ Caucasians which differs widely from reported ethnic backgrounds of western European SLE population[13]. This rises the need for an investigation of gender differences in SLE in a western European cohort. Likewise, the American cohort by Tan et al. includes only $60 \%$ Caucasians.

Thus, differences between male and female SLE described in the literature remain controversial and further investigation are needed. This motivated us to study in more detail gender differences in SLE using the first Swiss SLE cohort derived from different tertiary, secondary and primary care centers and lasting over 10 years. We took advantage of this prospective, multidisciplinary Swiss systemic lupus erythematosus cohort study (SSCS)[14] to address that question and compared SLE manifestations and the renal and cardiovascular outcome of male versus female patients.

\section{Methods}

All patients in this study were included in the Swiss SLE Cohort Study. This nationwide prospective cohort was established in 2007 as a collaboration between tertiary, secondary and primary care centers encompassing various medical specialties in Switzerland[14].

\section{Patients and Data}

Patients at least 16 years old with diagnosed SLE according to the updated American College of Rheumatology (ACR) criteria[15,16] and informed consent were continuously included into the cohort between 2007 and 2017 by their treating doctor. Patient data such as age, sex, ethnic background, family history of SLE, date of first SLE manifestation and date of diagnosis was collected at inclusion. The presence of all cumulative clinical manifestations defined by the updated ACR classification criteria of SLE prior to inclusion were reported. A follow up was conducted yearly and at disease flares by the patient's treating doctor. At inclusion and at each follow up laboratory values as serum haemoglobin, thrombocytes, creatinine, erythrocytes sedimentation rate were measured. Additionally at inclusion and every follow up medication, disease activity, need for renal replacement treatment and deaths of the patients were reported. Disease activity was measured with Systemic Lupus Erythematosus Disease Activity Index score with the Safety of Estrogens in Lupus Erythematosus modification (SELENA SLEDAI) 
score and physicians global assessment (PGA) score[14]. At least once during the follow up period the Systemic Lupus International Collaborating Clinics/American College of Rheumatology Damage Index (SLICC-SDI) was assessed for patients with over six months of disease duration. The SELENA SLEDAI[17] as well as the SLICC-SDI[18] are standardized scores to quantify disease activity of SLE or respectively the cumulative and irreversible organ damage and make comparisons between the patients in studies possible.

The estimated glomerular filtration rate (eGFR) was calculated by chronic kidney disease epidemiology collaboration equation (CKD-EPI)[19] based on serum creatinine values. The decision to perform a renal biopsy was left to the treating physician according to clinical practice. The performed renal biopsies were reported at patient's inclusion or at the next follow up. We analyzed the most recent available biopsy result of a patient. Lupus nephritis was either classified according to International Society of Nephrology and Renal Pathology Society (ISN/RPS)[20] or to World Health Organization (WHO) 1982 modified classification[21]. The medication was categorized in three groups: antimalarial drugs, systemic glucocorticoids and immunosuppressive agents other than glucocorticoids. We analyzed how many patients used one of the medication at least once during follow up period or at baseline.

\section{Outcomes}

We investigated the difference in renal outcome between the genders. Renal failure was defined as eGFR $<15 \mathrm{ml} / \mathrm{min} / 1.73 \mathrm{~m}^{2}$, initiation of renal replacement therapy, documented end stage renal disease in SLICC-SDI or doubling of serum creatinine. In order to adjust for age difference, disease duration, time from SLE manifestation to diagnosis, ethnic background, medication used during follow up period, eGFR at inclusion and SELENA-SLEDAI Score at inclusion cox regression for renal failure was used.

Furthermore, we analyzed the overall damage caused by SLE and the occurrence of cardiovascular events with the information provided by SLICC-SDI. A multivariate analysis was performed to control the gender differences in cardiovascular outcomes for confounders.

\section{Statistical analysis}

Data are presented as absolute numbers with percentages for categorical variables, as medians with $25 \%$ - and $75 \%$-quartiles for not normally distributed continuous variables or as means with standard deviation for normally distributed variables. Comparison between two groups was assessed by MannWhitney $U$ test for continuous variables and chi-square test or fisher's exact test for categorical variables. In the presented Tables 1-4 only univariate comparisons were displayed.

For categorical outcome variables such as SLE manifestations and cardiovascular outcomes a multivariate analysis was performed using multiple logistic regression. We included in the logistic regression models independed variables, which had a p-value $<0.1$ in the univariate comparison with the outcome variable. In a second step we checked for interactions between the significant, independent variables. The different models were compared by akaike information criterion (AIC) and the model with the lowest AIC was chosen. 
Renal failure curves were developed with cox regression. Patients were included in the cox regression at inclusion to the cohort and if no renal failure occurred during the follow up period, they were censored at their last follow up visit. We included gender, age at inclusion, disease duration, time from SLE manifestation to diagnosis, ethnic background, medication used during follow up period, eGFR at inclusion and SELENA-SLEDAI Score at inclusion in the cox regression model.

Overall mortality during the follow up period was analyzed using kaplan meier survival curves and compared with log rank test.

A p-value $<0.05$ was considered statistically significant. Statistical analysis was performed with IBM SPSS Statistics 23.0 statistical software package (SPSS Inc, Chicago, Illinois, USA).

All methods were carried out in accordance with relevant guidelines and regulations.

\section{Results}

\section{Patients' baseline characteristics}

We analyzed a total of 622 patients in our cohort, of which 529 (85\%) were female and 93 (15\%) were male. The female to male ratio was 5.7:1. The majority of patients were Caucasian ( $81 \%$ of females and $82 \%$ of males) and the ethnic background was comparable between males and females.

The median age at diagnosis was 33.1 years in women and 44.4 years in men, the difference was significant with a p-value $<0.001$. Men were significantly older at inclusion than women (48.2 versus 42.9 years, $p=0.002)$ and had a significantly shorter median disease duration at inclusion (2.2 versus 3.4 years, $p=0.018$ ). The median time between onset of symptoms and SLE diagnosis was 0.3 years in both genders. In total 49 patients ( $8 \%$ ) were lost to follow up of which 4 were males ( $4 \%$ of all males) and 45 were females $(9 \%)$.

Medication used during follow up period differed between the two groups: Significantly more males were treated at least once during the follow up period with immunosuppressant agents and oral corticosteroids than females (Table 1). The difference in the use of antimalarial medication was not significant. 
Table 1

Baseline characteristics of the study population

\begin{tabular}{|c|c|c|c|}
\hline Demographics & Women & Men & p-value ${ }^{a}$ \\
\hline Number of patients & $529(85 \%)$ & $93(15 \%)$ & \\
\hline Age at baseline (years) & $42.9(32.0,53.3)$ & $48.2(36.2,65.9)$ & 0.002 \\
\hline \multicolumn{4}{|l|}{ Ethnic background } \\
\hline - Caucasian & $416(81 \%)$ & $73(82 \%)$ & \\
\hline - African & $36(7 \%)$ & $6(7 \%)$ & \multirow[t]{3}{*}{0.995} \\
\hline - Asian & $44(9 \%)$ & $7(8 \%)$ & \\
\hline - Other & $19(4 \%)$ & $3(3 \%)$ & \\
\hline First degree relatives with SLE & $58(13 \%)$ & $8(11 \%)$ & 0.709 \\
\hline \multicolumn{4}{|l|}{ SLE Characteristics } \\
\hline Age at diagnosis (years) & $33.1(24.1,45.6)$ & $44.4(28.7,57.0)$ & $<0.001$ \\
\hline Time to diagnosis (years) & $0.3(0.0,2.0)$ & $0.3(0.0,1.7)$ & 0.956 \\
\hline Disease duration (years) & $3.4(0.8,10.2)$ & $2.2(0.4,7.2)$ & 0.018 \\
\hline Follow up time (years) & $2.0(0.0,4.9)$ & $2.0(0.0,4.9)$ & 0.815 \\
\hline \multicolumn{4}{|c|}{ Laboratory assessment at baseline } \\
\hline Haemoglobin $(\mathrm{g} / \mathrm{l})$ * & $123 \pm 22$ & $134 \pm 21$ & $<0.001$ \\
\hline Thrombocytes $(\mathrm{G} / \mathrm{L})$ * & $243 \pm 89$ & $226 \pm 88$ & 0.011 \\
\hline Serum creatinine $(\mu \mathrm{mol} / \mathrm{l})$ & $67(59,79)$ & $86(73,99)$ & $<0.001$ \\
\hline eGFR $\left(\mathrm{ml} / \mathrm{min} / 1.73 \mathrm{~m}^{2}\right)$ & $94(74,111)$ & $93(70,111)$ & 0.412 \\
\hline \multicolumn{4}{|l|}{ Medication ${ }^{b}$} \\
\hline Antimalarial medication & $414(79 \%)$ & $66(73 \%)$ & 0.217 \\
\hline Immunosuppressant agents & $302(57 \%)$ & $65(71 \%)$ & 0.021 \\
\hline Oral corticosteroids & $331(63 \%)$ & $70(76 \%)$ & 0.018 \\
\hline
\end{tabular}

Values were presented as medians with $25 \%$ and $75 \%$ quartile in brackets for continuous variables or as absolute values with percentages for categorical variables.

*Normally distributed continuous variables were presented as mean \pm standard deviation

${ }^{\text {a }}$ P-values $<0.05$ were considered statistically significant and written in bold. 
b Use of antimalarial medication, oral corticosteroids or immunosuppressants other than corticosteroids at baseline and/or at least once during the follow up period.

\section{Clinical and immunological manifestation}

At inclusion, men had a statistically significant lower median number of cumulative ACR criteria than women ( 4 versus 5 points, $p$-value $=0.007$ ). Women had significantly higher prevalence of dermatological manifestation, 403 women $(76 \%)$ versus 57 men $(61 \%)(p=0.005)$. Regarding the individual dermatological manifestation, only the difference in photosensitivity was significant. Arthritis was more common in women than in men, $392(74 \%)$ and $53(57 \%)$ respectively $(p=0.001)$. Women had a higher prevalence of psychosis with 34 women $(6 \%)$ versus one man $(1 \%)(p=0.047)$. There were no significant differences in other clinical or immunological manifestations (Table 2).

In multivariate analysis for arthritis we included gender, anti-Sm antibodies, anti-dsDNA antibodies, eGFR at inclusion, SELENA SLEDAI Score at inclusion, oral corticosteroids at inclusion, age at diagnosis and disease duration at inclusion in the model. Gender, anti-Sm antibodies, oral corticosteroids, eGFR and disease duration were all significantly associated with arthritis. Male gender had an odds ratio (OR) of 0.31 for arthritis with a $95 \%$ confidence interval $(95 \%-\mathrm{Cl}) 0.16-0.59, \mathrm{p}<0,001$. After inclusion of an interaction factor between gender and disease duration, the effect of disease duration was reduced but remained significant (supplementary table 1 ).

In multivariate analysis for dermatological manifestations, we included gender, disease duration at inclusion, PGA at inclusion and anti-Sm antibodies. Only gender and disease duration were significant. When including only gender and disease duration in the model, only male gender was significant with an OR of $0.51(95 \%-\mathrm{Cl} 0.32-0.82, \mathrm{p}=0.005)$ (supplementary table 2$)$. After inclusion of the interaction factor between gender and disease duration, the effect of gender was not significant any more, but the overall multivariate model had a higher AIC showing no overall improvement of the model.

In the multivariate model for photosensitivity, we included gender, ethnic background, age at diagnosis, disease duration at inclusion, PGA at inclusion, anti-dsDNA antibodies and antimalarial medication at inclusion. Gender, ethnic background, anti-dsDNA antibodies, PGA, antimalarial medication and disease duration were all significant. Male gender had an OR of $0.41(95 \%-\mathrm{Cl} 0.24-0.70, \mathrm{p}=0.001)$ (supplementary table 3). The interaction factor between gender and disease duration did not significantly change the results nor was the overall model improved. 
Table 2

SLE manifestations prior to inclusion according to ACR criteria

\begin{tabular}{|llll|}
\hline & Women & Men & p-value $^{\text {a }}$ \\
\hline Number of total ACR criteria (points) & $5(4,6)$ & $4(4,6)$ & 0.007 \\
\hline Clinical manifestations & & & \\
\hline Dermatological disorder & $403(76 \%)$ & $57(61 \%)$ & 0.005 \\
\hline Photosensitivity & $269(51 \%)$ & $26(28 \%)$ & $<0.001$ \\
\hline Malar rash & $208(39 \%)$ & $28(30 \%)$ & 0.105 \\
\hline Discoid rash & $112(21 \%)$ & $14(15 \%)$ & 0.208 \\
\hline Oral and nasal ulcers & $158(30 \%)$ & $21(23 \%)$ & 0.172 \\
\hline Arthritis & $392(74 \%)$ & $53(57 \%)$ & 0.001 \\
\hline Serositis & $142(27 \%)$ & $32(34 \%)$ & 0.168 \\
\hline Pleuritis & $107(20 \%)$ & $26(28 \%)$ & 0.102 \\
\hline Pericarditis & $96(18 \%)$ & $16(17 \%)$ & 0.885 \\
\hline Renal disorder & $197(37 \%)$ & $39(42 \%)$ & 0.419 \\
\hline Haematologic disorder & $317(60 \%)$ & $60(64 \%)$ & 0.490 \\
\hline Central nervous system involvement & $46(9 \%)$ & $7(8 \%)$ & 0.842 \\
\hline Psychosis & $34(6 \%)$ & $1(1 \%)$ & 0.047 \\
\hline Seizures & $22(4 \%)$ & $6(6 \%)$ & 0.290 \\
\hline Immunological manifestations & & & \\
\hline Anti-nuclear antibodies & $516(98 \%)$ & $88(95 \%)$ & 0.076 \\
\hline Anti-dsDNA antibodies & $338(64 \%)$ & $64(69 \%)$ & 0.412 \\
\hline Anti-Sm antibodies & $231(44 \%)$ & $34(37 \%)$ & 0.210 \\
\hline Antiphospholipid antibodies & & & \\
\hline & & & \\
\hline
\end{tabular}

Values were presented as medians with $25 \%$ and $75 \%$ quartile in brackets for continuous variables or as absolute values with percentages for categorical variables.

a P-values $<0.05$ were considered statistically significant and written in bold.

\section{Disease activity}

There was no significant difference in disease activity at baseline between the genders. The SELENASLEDAI Score, PGA and erythrocyte sedimentation rate (ESR) were all similar (Table 3). 


\section{Overall damage outcome}

We compared the most recent SLICC-SDI Score between the two groups which was available for 475 women and 83 men. In these two groups men were older at the time of SLICC-SDI Score (52.4 versus 45.7 years, $p=0.001)$. Women had a significantly higher disease duration at the time of the SLICC-SDI (6.9 versus 5.5 years, $p=0.026$ ). Men tended to have higher SLICC-SDI Score (Table 3 ). 
Table 3

Damage accrual and disease activity

\begin{tabular}{|llll|}
\hline & Women & Men & p-value $^{\text {a }}$ \\
\hline Total Patients & 475 & 83 & \\
\hline Age at SLICC-SDI (years) & $45.7(35.0,57.1)$ & $52.4(40.7,68.5)$ & 0.001 \\
\hline Disease duration at SLICC-SDI (years) & $6.9(3.0,13.6)$ & $5.5(2.0,10.7)$ & 0.026 \\
\hline Time between inclusion and SLICC-SDI (years) & $2.0(0.0,4.9)$ & $1.4(0.0,4.6)$ & NS \\
\hline SLICC-SDI & & & \\
\hline Score (points) & $0(0,2)$ & $1(0,3)$ & 0.072 \\
\hline Cerebrovascular insult & $27(6 \%)$ & $6(7 \%)$ & 0.789 \\
\hline Any renal complication & $52(11 \%)$ & $10(12 \%)$ & 0.709 \\
\hline - Chronic kidney disease & $44(9 \%)$ & $9(11 \%)$ & 0.051 \\
\hline - Proteinuria & $13(3 \%)$ & $2(2 \%)$ & 0.658 \\
\hline - ESRD & $13(27 \%)$ & $3(4 \%)$ & 0.718 \\
\hline Any cardiac complication & $57(12 \%)$ & $20(24 \%)$ & 0.006 \\
\hline - Coronary vascular disease & $20(4 \%)$ & $14(17 \%)$ & $<0.001$ \\
\hline - Myocardial infarctus & $11(2 \%)$ & $8(10 \%)$ & 0.003 \\
\hline - Cardiomyopathy & $21(4 \%)$ & $5(6 \%)$ & 0.568 \\
\hline - Valvular disease & $26(5 \%)$ & $7(8 \%)$ & 0.310 \\
\hline - Chronic pericarditis & $7(1 \%)$ & $0(0 \%)$ & 0.601 \\
\hline Any peripher vascular complication & $13(3 \%)$ & $2(2 \%)$ & 1.0 \\
\hline - Claudicatio & $6(1 \%)$ & $2(2 \%)$ & 0.339 \\
\hline - Minor tissue loss & $7(1 \%)$ & $0(0 \%)$ & 0.601 \\
\hline - Significant tissue loss & $3(1 \%)$ & $0(0 \%)$ & 1.0 \\
\hline Disease activity & & $4(2,9)$ & 0.496 \\
\hline SELENA SLEDAI at baseline (points) & $4(2,8)$ & & \\
\hline
\end{tabular}




\begin{tabular}{|llll|}
\hline & Women & Men & p-value $^{\text {a }}$ \\
\hline PGA at baseline (points) & & & \\
- inactive & $243(46 \%)$ & $41(44 \%)$ & \\
- moderately active & $184(35 \%)$ & $35(38 \%)$ & 0.484 \\
- active & $87(16 \%)$ & $12(13 \%)$ & \\
- very active & $15(3 \%)$ & $5(5 \%)$ & \\
ESR $(\mathrm{mm} / \mathrm{h})$ at baseline & $14(7,33)$ & $16(7,35)$ & 0.861 \\
\hline
\end{tabular}

Values were presented as medians with $25 \%$ and $75 \%$ quartile in brackets for continuous variables or as absolute values with percentages for categorical variables.

a P-values $<0.05$ were considered statistically significant and written in bold.

\section{Lupus nephritis}

Renal disease occurred in 39 men (42\%) and in 197 women (37\%). This difference was not significant. Results from renal biopsies were available for 20 men and 89 women. The most common type of lupus nephritis without regard to classification system was class IV in 11 men (55\%) and 35 women (39\%), followed by class III in 6 men (30\%) and 18 women (20\%). The overall distribution of lupus nephritis classes was similar between genders (Table 4). 
Table 4

Lupus nephritis classes of patients with renal involvement

\begin{tabular}{|c|c|c|c|}
\hline & Women & Men & p-value a \\
\hline Renal biopsy available & 89 & 20 & \\
\hline \multicolumn{4}{|l|}{ Classification system } \\
\hline - ISN/RPS 2003 & $37(42 \%)$ & $10(50 \%)$ & \multirow[t]{3}{*}{0.833} \\
\hline - WHO modified 1982 & $15(17 \%)$ & $2(10 \%)$ & \\
\hline - Unknown & $37(42 \%)$ & $8(40 \%)$ & \\
\hline \multicolumn{4}{|l|}{ Lupus nephritis class* } \\
\hline \multicolumn{4}{|c|}{ WHO modified 1982 classification } \\
\hline-1 & $0(0 \%)$ & $0(0 \%)$ & \multirow[t]{6}{*}{0.839} \\
\hline$-I I$ & $2(13 \%)$ & $0(0 \%)$ & \\
\hline- - III & $3(20 \%)$ & $0(0 \%)$ & \\
\hline$-\mathrm{IV}$ & $9(60 \%)$ & $2(100 \%)$ & \\
\hline$-V$ & $1(7 \%)$ & $0(0 \%)$ & \\
\hline$-\mathrm{VI}$ & $0(0 \%)$ & $0(0 \%)$ & \\
\hline \multicolumn{4}{|l|}{ Lupus nephritis class* } \\
\hline \multicolumn{4}{|c|}{ ISN/RPS 2003 classification } \\
\hline-1 & $0(0 \%)$ & $0(0 \%)$ & \multirow[t]{8}{*}{0.562} \\
\hline 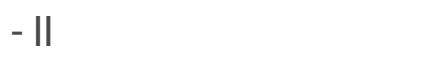 & $6(16 \%)$ & $0(0 \%)$ & \\
\hline$-\mathrm{III}$ & $8(22 \%)$ & $4(40 \%)$ & \\
\hline$-\mathrm{IV}$ & $15(41 \%)$ & $5(50 \%)$ & \\
\hline$-V$ & $4(11 \%)$ & $1(10 \%)$ & \\
\hline$-\mathrm{VI}$ & $0(0 \%)$ & $0(0 \%)$ & \\
\hline$-V+I I I$ & $3(8 \%)$ & $0(0 \%)$ & \\
\hline$-V+I V$ & $1(3 \%)$ & $0(0 \%)$ & \\
\hline \multicolumn{4}{|l|}{ Lupus nephritis class* } \\
\hline unknown classification & & & \\
\hline
\end{tabular}




\begin{tabular}{|llll|}
\hline & Women & Men & p-value $^{\text {a }}$ \\
\hline - I & $0(0 \%)$ & $0(0 \%)$ & 0.788 \\
- II & $6(15 \%)$ & $0(0 \%)$ & \\
- III & $7(19 \%)$ & $2(25 \%)$ & \\
- IV & $11(30 \%)$ & $4(50 \%)$ \\
- V & $7(19 \%)$ & $1(13 \%)$ \\
- VI & $0(0 \%)$ & $0(0 \%)$ \\
- V + III & $4(11 \%)$ & $1(13 \%)$ \\
- V + IV & $2(5 \%)$ & $0(0 \%)$ \\
\hline
\end{tabular}

Lupus nephritis classes of most recent available renal biopsy of patients with renal disorder displayed according to the used classification system.

Values were presented as absolute values with percentages.

${ }^{\text {a }}$ P-values $<0.05$ were considered statistically significant.

\section{Renal outcome}

We compared the incidence of renal failure between genders using cox regression model. Data of renal outcome was available for in total 495 patients ( 416 females and 79 males), 36 of them had renal failure. We included gender, age at inclusion, ethnicity, disease duration at inclusion, time from disease manifestation to diagnosis, medication during follow up, eGFR and SELENA SLEDAI at inclusion in the cox regression model (Table 5). Male gender had a significantly increased hazard ratio of 2.5 with a $95 \%$ Cl 1.1-6.0 and a p-value of 0.038 (Fig. 1). 
Table 5

Cox regression model for renal failure

\begin{tabular}{|lllll|}
\hline & B & SE (B) & p-value & HR \\
\hline Gender & 0.925 & 0.445 & 0.038 & 2.521 \\
\hline Antimalarial medication ${ }^{\text {a }}$ & 0.156 & 0.424 & 0.712 & 1.169 \\
\hline Corticosteroids $^{\text {a }}$ & -0.308 & 0.494 & 0.533 & 0.735 \\
\hline Immunosuppressants $^{\text {a }}$ & 0.867 & 0.518 & 0.094 & 2.380 \\
\hline SELENA SLEDAl & 0.013 & 0.023 & 0.576 & 1.013 \\
\hline Age at inclusion & -0.023 & 0.013 & 0.081 & 0.977 \\
\hline Time to diagnosis & 0.039 & 0.020 & 0.054 & 1.040 \\
\hline eGFR at inclusion & -0.039 & 0.006 & $<0.001$ & 0.962 \\
\hline Disease duration & 0.029 & 0.018 & 0.107 & 1.029 \\
\hline Ethnic background & & & 0.967 & \\
\hline Ethnic background(1) & -7.052 & 118.300 & 0.952 & 0.001 \\
\hline Ethnic background(2) & 0.603 & 134.605 & 0.996 & 1.828 \\
\hline Ethnic background(3) & 4.312 & 94.433 & 0.964 & 74.586 \\
\hline Ethnic background(4) & 0.334 & 84.726 & 0.997 & 1.396 \\
\hline Ethnic background(5) & -2.140 & 122.609 & 0.986 & 0.118 \\
\hline
\end{tabular}

Table of estimates of all variables included in the cox regression model for renal failure. Gender $(0=$ female, $1=$ male), medication during follow up, SELENA SLEDAI Score at baseline, age in years at baseline, disease duration in years at baseline, time from SLE manifestation to SLE diagnosis in years and ethnic background were included in the model. $\mathrm{B}=$ regression coefficient, $\mathrm{SE}=$ standard error for $\mathrm{B}$, $\mathrm{HR}=$ hazard ratio.

a Use of antimalarial medication, oral corticosteroids or immunosuppressants other than corticosteroids at baseline and/or at least once during the follow up period.

\section{Cardiovascular outcome}

In the most recent SLICC-SDI Score men had significantly higher rates of coronary arterial disease (CAD) and myocardial infarction. 14 men $(17 \%)$ versus 20 women $(4 \%)$ had CAD $(p<0.001)$. Eight men $(10 \%)$ versus 11 women $(2 \%)$ had myocardial infarction $(p=0.003)$. Cerebrovascular, peripheral vascular and cardiac complications were not significantly different (Table 3). The SLICC SDI Score was available of males 83 and females 475 (Table 3). 
We performed a multiple logistic regression for CAD in which we included gender, age at time of the SLICC-SDI, total SLICC-SDI Score, ESR at inclusion, SELENA SLEDAI at inclusion, eGFR at inclusion, pericarditis at inclusion and proteinuria documented in the SLICC-SDI Score. Gender, age and total SLICCSDI Score were significant (Table 6). Gender had an OR of 5.6 (95\%-Cl 2.3-13.7, p< 0.001). After inclusion of an interaction factor between gender and age, the effect of gender was less significant. However the overall model had a higher AIC compared to the model without the interaction factor.

We included gender, age at time of the SLICC-SDI, total SLICC-SDI Score, ESR at inclusion, eGFR at inclusion, chronic kidney disease (CKD) and proteinuria documented in the SLICC-SDI Score in the multiple logistic regression model for myocardial infarction. Gender, age, total SLICC SDI Score and CKD were significant (Table 7). Male gender had an OR of $8.3(95 \%-\mathrm{Cl} 2.1-32.6, \mathrm{p}=0.002)$. After inclusion of the interaction factor between age and gender, the effect of gender was no longer significant. However, the model with the interaction factor had a higher AIC compared to the model without the interaction factor, suggesting its inferiority.

Table 6

Logistic regression for coronary artery disease

\begin{tabular}{|lllll|}
\hline & $\boldsymbol{\beta}$ & SE $(\boldsymbol{\beta})$ & p-value & $\boldsymbol{B}$ \\
\hline Gender & 1.720 & 0.458 & $<0.001$ & 5.582 \\
\hline Age & 0.037 & 0.014 & 0.007 & 1.038 \\
\hline Total SLICC-SDI Score & 0.468 & 0.070 & $<0.001$ & 1.598 \\
\hline Constant & -6.499 & 0.875 & $<0.001$ & 0.002 \\
\hline
\end{tabular}

Table of estimates of the logistic regression model for coronary artery disease. Gender (male $=1$, female $=0$ ), age in years at time of the SLICC-SDI Score and total SLICC-SDI Score were included in the model. $\beta$ $=$ regression coefficient, $S E(\beta)=$ standard error $\operatorname{for} \beta, B=$ odds ratio, age = age in years at the time of the SLICC-SDI score.

Table 7

Logistic regression for myocardial infarction

\begin{tabular}{|lllll|}
\hline & $\boldsymbol{\beta}$ & SE $(\boldsymbol{\beta})$ & p-value & $\mathbf{B}$ \\
\hline Gender & 2.117 & 0.698 & 0.002 & 8.305 \\
CKD & -2.463 & 1.076 & 0.022 & 0.085 \\
Age & 0.049 & 0.023 & 0.033 & 1.050 \\
\hline Total SLICC-SDI Score & 0.751 & 0.133 & $<0.001$ & 2.119 \\
\hline Constant & -9.038 & 1.644 & $<0.001$ & $<0.001$ \\
\hline
\end{tabular}

Table of estimates of the logistic regression model for myocardial infarction. Gender (male $=1$, female $=$ 0), age in years at time of the SLICC-SDI Score, total SLICC-SDI Score and chronic kidney disease (CKD) 
documented in the SLICC-SDI Score were included in the model. $\beta=$ regression coefficient, SE $(\beta)=$ standard error for $\beta, B=$ odds ratio.

\section{Mortality}

In total $22(4 \%)$ patients died during the follow up period of which $14(3 \%)$ were women and $8(9 \%)$ were men. The deaths of 6 patients were related to SLE. The causes of death in the other patients were infection, cerebrovascular accidents, end stage renal disease, heart insufficiency, brainstem vasculitis and in one case myocardial rupture due to bacterial infection and myocardial infarction.

Kaplan Meier analysis showed a significantly worse survival in men with a p-value of 0.005 . Men had an estimated mean survival time of 8.3 years and women 9.4 years. After 5 years estimated cumulative survival was $83 \%$ for men and $95 \%$ for women (Fig. 2).

\section{Discussion}

We analyzed gender differences in 529 women and 93 men of the observational, prospective Swiss systemic lupus erythematosus cohort study and found significant differences between male and female SLE regarding clinical manifestations and renal and cardiovascular outcome.

We found a significantly higher risk in males for cardiovascular complications such as myocardial infarction with an OR of 8.3 and CAD with an OR of 5.6. Age was as well a significant risk factor for cardiovascular outcome. Our multivariate models suggests that an age-dependent gender difference may exist and explain partially the gender difference in cardiovascular outcome. Nevertheless, adding the interaction factor between gender and age did not improve the multivariate model. The model without the interaction factor was better by comparison of the AIC and showed a significant association between gender and CAD and myocardial infarction. This suggests that gender could be a risk factor for cardiovascular outcome independently of age. Similarly, in the mentioned LUMINA study men had a higher risk for any cardiovascular damage compared to women with an OR of 3.6[7]. This is in line with several other studies on SLE in males[8,22-24].

It is known that in general population men have a higher cardiovascular risk than women[25]. The American heart association reported that women and men aged between 40 and 59 years had a prevalence of $1.8 \%$ versus $3.3 \%$ for myocardial infarction and $5.9 \%$ versus $6.3 \%$ for coronary heart disease[26]. Our observed risk difference between genders in patients with SLE seems to be higher than the reported risk difference in general population.

Previous studies suggest a higher risk for patients with SLE to have any cardiovascular disease which can not only be explained by traditional cardiovascular risk factors[6]. Non traditional risk factors seem to have as well a big impact such as systemic inflammation, systemic disease and medication related risk factors[6,7]. We recently showed that serum calcification propensity measured by the T50 score test was closely associated with disease activity, suggesting that non traditional, lupus-specific risk factors contribute considerably to premature atherosclerosis and therefore cardiovascular events [27]. 
We found a worse renal outcome with a higher hazard ratio for renal failure in men than in women which is consistent with the large US-cohort of Tan et al. and other smaller cohorts who found as well significantly higher rates of renal insufficiency and renal failure in men and[8,22,28]. In contrast, no differences were found in the rate of renal failure both in a recent study of a large low income USpopulation with lupus nephritis[29], as well as in a rather small cohort with 30-years follow[10]. Differences in ethnicity, sample size, socioeconomic status, follow up period and definitions of renal failure may explain these controversial results. In the review by Murphy et al. it was suggested that these differences may be biased by the recruitment process, showing more renal involvement in studies held in nephrology clinics[9]. In our study however, patients were recruited in different specialty clinics which may avoid the specialty-based recruitment bias.

The age at diagnosis in this cohort was significantly higher in men than women which is consistent with several previous studies[8,22,30,31]. Population based studies in France and Germany showed that the incidence rates have a peak in a much younger age in women than in men[32,33]. In other studies however, the age at diagnosis was similar between the genders $[5,10,23,34]$. This controversy could be due to ethnic and geographic factors which differ among the studies, since previously reported data seems to show higher age in European men[35].

We were expecting a longer delay from first disease manifestation to SLE diagnosis in men, possibly linked to its rarity and postulated different clinical manifestation which would lead to a delayed consideration of SLE in the diagnostic process of these patients[36]. However, in our cohort time to diagnosis was similar between genders. Therefore, a delay in diagnosis in men may not explain a possible greater burden of disease and damage. In a Latin American cohort the time to diagnosis was even significantly shorter in men, suggesting a faster progression to overt SLE[23].

Regarding clinical manifestation of SLE, men were less likely to develop dermatological manifestations, arthritis and psychosis. In the multivariate models, female gender remained a risk factor for development of photosensitivity, any dermatological manifestations and arthritis. There was an interaction between disease duration and gender in the multivariate model for dermatological manifestations. However in the multivariate model for arthritis and photosensitivity male gender remained unchanged and significantly associated with arthritis or photosensitivity regardless of the inclusion of the interaction factor with disease duration. A multivariate analysis for psychosis was not performed due to the very small number of patients, especially in men. Our results are in line with literature where men are less likely to have skin involvement $[8,9,23,35]$. Our findings regarding arthritis are consistent with multiple studies, including the Latin American cohort[23,35,37]. Other studies, however, showed no differences in arthritis[8,10,30,31,34], but two of them found a higher prevalence of arthralgia in women[8,34]. Generally, our study suggests that men have the same spectrum of disease manifestations, but a possibly a difference in prevalence of certain manifestations than women.

The prevalence of renal involvement in men remains controversial.[9] A higher prevalence of renal involvement was described in some studies, including the large cohort by Tan et al[8,23,31,34]. Our study 
did not show a higher prevalence of renal involvement in men, which is consistent with a small Spanish[30] and large multi-ethnic US cohort[5].

We did not find any significant difference between the genders in immunological manifestations. In contrast, a recent meta-analysis showed significantly higher prevalence of anti-dsDNA in men, higher prevalence of lupus anticoagulant and ANA in women and lower levels of complement factor 3 (C3) in women[35]. However, similar to our results the LUMINA study found no significant differences in immunological manifestations besides a higher prevalence of lupus anticoagulant in women[5]. The above mentioned Latin American cohort found no differences apart from significantly higher prevalence of Anti-cardiolipin antibodies and low C3 levels in men[23].

Regardless the gender difference in cardiovascular damage, the overall damage measured by SLICC-SDI Score was similar between genders which is in line with previous studies[23]. There was no difference in disease activity at inclusion as well.

The mortality rate was significantly higher in men than women in this cohort, which is found in the 1981 study of Wallace et al. and more recent studies as well[4,36].

Our work is the first gender study in a western European population. To date the larger studies that have examined sex differences have only a small proportion of Caucasians, and their results are therefore poorly generalizable to western European populations. Data of SLE from France show a prevalence of $47 / 100000$ [33], which means our cohort of 622 patients would account for approximately $15 \%$ of all SLE patients in Switzerland. The physicians recruiting patients for the cohort come from various disciplines, including dermatology, rheumatology, nephrology, immunology and internal medicine. In addition, these physicians do not all practice in a university hospital, but also in smaller regional hospitals and a private practice. This contributes significantly to the representativeness of our study. Furthermore, considering the impact of ethnicity in SLE disease course[12], our results provide information for clinicians in other western European populations of which other SLE cohorts reported similar ethnical background[13].

Our study has some limitations. The patients in our cohort have been included at different times of their disease course, some of them right after diagnosis, others after a long disease duration. This can influence the longitudinal findings. The age between the two compared groups was significantly different at baseline, which can confound the data as well. To counteract this, we included age and disease duration in our multivariate models for SLE manifestations, cardiovascular and renal outcomes. As in all cohort studies we cannot control our results for unknown or unmeasured confounders. Furthermore, since most patients had only once an evaluation of the SLICC-SDI Score, it allowed us only to uphold cross sectional information of cardiovascular events and overall damage.

The reasons for the observed gender differences in SLE-related outcomes are still unknown. Multiple reasonable hypotheses exist such as hormonal, sex chromosomal theories and intrauterine selection, but none of them achieve to fully explain the observed differences[36]. For example, sex hormonal hypothesis is supported by murine models where female hormones seem to increase risk of SLE and disease flares, 
while androgens seem to be beneficial. However, clinical trials could not confirm completely these effects on SLE in humans[36]. The sex chromosome theory is based on the finding that Klinefelter's syndrome has a strong association with SLE, indicating that two X-chromosomes increase the risk of lupus by 10fold[36]. However, further investigations are needed to understand this association. Additionally, it has been proposed that behavioral factors have an influence as well, leading to lower likelihood of men with a mild disease to consult a doctor than women which can cause a statistical bias[23].

\section{Conclusion}

Our study investigated gender differences in SLE in a large national cohort and found significant differences. This was the first of its kind in a western European population. In our study, men were less likely to have arthritis and dermatological manifestations, especially photosensitivity than women. Regarding outcome, they had a higher risk for renal failure and male gender was a significant risk factor

for cardiovascular events. Further research in this area is needed and could lead to a better understanding of the etiology of SLE in general and help provide gender specific treatment options.

\section{Abbrevations}

SLE = systemic lupus erythematosus

SLICC-SDI = Systemic Lupus International Collaborating Clinics/American College of Rheumatology Damage Index

$\mathrm{CAD}=$ coronary artery disease

$E S R D=$ end stage renal disease

$\mathrm{ESR}=$ erythrocyte sedimentation rate

ANA $=$ anti-nuclear antibodies

Anti-dsDNA = anti-double stranded DNA

SSCS $=$ Swiss SLE Cohort Study

$\mathrm{ACR}=$ American College of Rheumatology

ISN/RPS = International Society of Nephrology and Renal Pathology Society

WHO = World Health Organization

SELENA SLEDAI = Systemic Lupus Erythematosus Disease Activity Index score with the Safety of Estrogens in Lupus Erythematosus modification 
eGFR = estimated glomerular filtration rate

CKD-EPI = chronic kidney disease epidemiology collaboration equation

$\mathrm{CKD}=$ chronic kidney disease

$\mathrm{SD}=$ standard deviation

$\mathrm{SE}=$ standard error

PGA = physicians global assessment

$\mathrm{C} 3=$ complement factor 3

$95 \%-\mathrm{Cl}=95 \%$ Confidence interval

AIC = Akaike information criterion

$\mathrm{OR}=$ odds ratio

\section{Declarations}

\section{Ethics approval and consent to participate}

All data was collected from the Swiss SLE Cohort Study which was approved by the ethics review boards of all participating institutions (i.e., Commission catonale d'éthique de la recherche sur l'être humain Vaud, Kantonale Ethikkommission Bern, Ethikkommission Nordwest- und Zentralschweiz, Commission cantonale d'éthique de la recherche Genève, Ethikkommission Ostschweiz, Kantonale Ethikkommission Zürich) and all patients gave written informed consent. Informed consent was obtained from the parents/ legally authorized representatives from two study participants, who were 16.5 respectively 17.9 years of age at inclusion in the study.

\section{Consent for publication}

Not applicable

\section{Availability of data and materials}

The data that support the findings of this study are available from the Swiss SLE Cohort Study but are not publicly available. Data are however available from the authors upon reasonable request and with permission of the Swiss SLE Cohort Study.

\section{Competing interests}

The authors have declared no conflicts of interest. 


\section{Funding}

This work was supported by the Gebert-Rüf Foundation [grant number GRS-027/07 to SSCS].

\section{Authors' contributions}

JM analyzed the data, performed the statistical analysis. JM, SD and UH interpreted the results and wrote the manuscript. All authors read and approved the final manuscript.

\section{Acknowledgement}

The authors would like to thank all members of the Swiss Systemic Lupus Erythematosus Cohort Study who have contributed patient data: Eric Dayer (Clinical Immunology and Allergy, Sion Hospital), Ute Eisenberger (Essen), Thomas Hauser (Clinical Immunology and Allergy, Zurich), Annette Leimgruber (Clinical Immunology and Allergy, Lausanne), Petra Otto (Rheumatology, Kantonsspital St Gallen), Thomas Stoll (Rheumatology, Kantonsspital Schaffhausen).

\section{References}

1. Cervera R, Khamashta M, Hughes G. The Euro-lupus project: epidemiology of systemic lupus erythematosus in Europe. Cervera R, Tincani A, editors. Lupus. 2009;18:869-74.

2. SAMPAIO-BARROS PD, BORTOLUZZO AB, MARANGONI RG, ROCHA LF, DEL RIO APT, SAMARA AM, et al. Survival, Causes of Death, and Prognostic Factors in Systemic Sclerosis: Analysis of 947 Brazilian Patients. J Rheumatol. 2012;39:1971.

3. Ngian G-S, Stevens W, Prior D, Gabbay E, Roddy J, Tran A, et al. Predictors of mortality in connective tissue disease-associated pulmonary arterial hypertension: a cohort study. Arthritis Res Ther. 2012;14:R213.

4. Wallace DJ, Podell T, Weiner J, Klinenberg JR, Forouzesh S, Dubois EL. Systemic Lupus Erythematosus-Survival Patterns: Experience With 609 Patients. JAMA. 1981;245:934-8.

5. Andrade RM, Alarcón GS, Fernández M, Apte M, Vilá LM, Reveille JD, et al. Accelerated damage accrual among men with systemic lupus erythematosus: XLIV. Results from a multiethnic US cohort. Arthritis Rheum. 2007;56:622-30.

6. Roman MJ, Shanker B-A, Davis A, Lockshin MD, Sammaritano L, Simantov R, et al. Prevalence and Correlates of Accelerated Atherosclerosis in Systemic Lupus Erythematosus. N Engl J Med. 2003;349:2399-406.

7. Pons-Estel GJ, Gonzalez LA, Zhang J, Burgos PI, Reveille JD, Vila LM, et al. Predictors of cardiovascular damage in patients with systemic lupus erythematosus: data from LUMINA (LXVIII), a multiethnic US cohort. Rheumatology. 2009;48:817-22.

8. Tan TC, Fang H, Magder LS, Petri MA. Differences between Male and Female Systemic Lupus Erythematosus in a Multiethnic Population. J Rheumatol. 2012;39:759-69. 
9. Murphy G, Isenberg D. Effect of gender on clinical presentation in systemic lupus erythematosus. Rheumatology. 2013;52:2108-15.

10. Renau A, Isenberg D. Male versus female lupus: a comparison of ethnicity, clinical features, serology and outcome over a 30 year period. Lupus. 2012;21:1041-8.

11. Voulgari PV, Katsimbri P, Alamanos Y, Drosos AA. Gender and age differences in systemic lupus erythematosus. A study of 489 Greek patients with a review of the literature. Lupus. 2002;11:722-9.

12. Fernández M, Alarcón GS, Calvo-alén J, Andrade R, McGwin G, Vilá LM, et al. A multiethnic, multicenter cohort of patients with systemic lupus erythematosus (SLE) as a model for the study of ethnic disparities in SLE. Arthritis Care Res. 2007;57:576-84.

13. Cervera R, Doria A, Amoura Z, Khamashta M, Schneider M, Guillemin F, et al. Patterns of systemic lupus erythematosus expression in Europe. Autoimmun Rev. 2014;13:621-9.

14. Ribi C, Trendelenburg M, Gayet-Ageron A, Cohen C, Dayer E, Eisenberger U, et al. The Swiss Systemic lupus erythematosus Cohort Study (SSCS) - cross-sectional analysis of clinical characteristics and treatments across different medical disciplines in Switzerland. Swiss Med Wkly. 2014;144:w13990.

15. Tan EM, Cohen AS, Fries JF, Masi AT, Mcshane DJ, Rothfield NF, et al. The 1982 revised criteria for the classification of systemic lupus erythematosus. Arthritis Rheum. 1982;25:1271-7.

16. Hochberg MC. Updating the American College of Rheumatology revised criteria for the classification of systemic lupus erythematosus. Arthritis Rheum. 1997;40:1725.

17. Petri M, Kim MY, Kalunian KC, Grossman J, Hahn BH, Sammaritano LR, et al. Combined Oral Contraceptives in Women with Systemic Lupus Erythematosus. N Engl J Med. 2005;353:2550-8.

18. Gladman DD, Goldsmith CH, Urowitz MB, Bacon P, Fortin P, Ginzler E, et al. The Systemic Lupus International Collaborating Clinics/American College of Rheumatology (SLICC/ACR) Damage Index for Systemic Lupus Erythematosus International Comparison. J Rheumatol. 2000;27:373-6.

19. Levey AS, Stevens LA, Schmid CH, Zhang Y (Lucy), Castro AF, Feldman HI, et al. A New Equation to Estimate Glomerular Filtration Rate. Ann Intern Med. 2009;150:604.

20. Weening JJ, D'Agati VD, Schwartz MM, Seshan SV, Alpers CE, Appel GB, et al. The Classification of Glomerulonephritis in Systemic Lupus Erythematosus Revisited. J Am Soc Nephrol. 2004;15:241-50.

21. Churg J, Bernstein J, Glassock RJ. Renal disease: classification and atlas. 1: Renal disease: classification and atlas of glomerular diseases. 2. ed. New York: Igaku-Shoin; 1995.

22. Crosslin KL, Wiginton KL. Sex Differences in Disease Severity Among Patients With Systemic Lupus Erythematosus. Gend Med. 2011;8:365-71.

23. Garcia MA, Marcos JC, Marcos Al, Pons-Estel BA, Wojdyla D, Arturi A, et al. Male systemic lupus erythematosus in a Latin-American inception cohort of 1214 patients. Lupus. 2005;14:938-46.

24. Urowitz MB, Gladman D, Ibañez D, Bae SC, Sanchez-Guerrero J, Gordon C, et al. Atherosclerotic vascular events in a multinational inception cohort of systemic lupus erythematosus. Arthritis Care Res. 2010;62:881-7. 
25. Kappert K, Böhm M, Schmieder R, Schumacher H, Teo K, Yusuf S, et al. Impact of Sex on Cardiovascular Outcome in Patients at High Cardiovascular RiskClinical Perspective: Analysis of the Telmisartan Randomized Assessment Study in ACE-Intolerant Subjects With Cardiovascular Disease (TRANSCEND) and the Ongoing Telmisartan Alone and in Combination With Ramipril Global End Point Trial (ONTARGET). Circulation. 2012;126:934-41.

26. Mozaffarian D, Benjamin EJ, Go AS, Arnett DK, Blaha MJ, Cushman M, et al. Heart Disease and Stroke Statistics-2016 Update: A Report From the American Heart Association. Circulation. 2016;133:e38-360.

27. Dahdal S, Devetzis V, Chalikias G, Tziakas D, Chizzolini C, Ribi C, et al. Serum calcification propensity is independently associated with disease activity in systemic lupus erythematosus. PLOS ONE. 2018;13:e0188695.

28. Resende AL, Titan SM, Barros RT, Woronik V. Worse renal outcome of lupus nephritis in male patients: a case-control study. Lupus. 2011;20:561-7.

29. Feldman CH, Broder A, Guan H, Yazdany J, Costenbader KH. Sex Differences in Health Care Utilization, End-Stage Renal Disease, and Mortality Among Medicaid Beneficiaries With Incident Lupus Nephritis. Arthritis Rheumatol. 2018;70:417-26.

30. Gómez J, Suárez A, López P, Mozo L, Díaz JB, Gutiérrez C. Systemic Lupus Erythematosus in Asturias, Spain: Clinical and Serologic Features. Medicine (Baltimore). 2006;85:157-68.

31. Alonso MD, Martínez-Vázquez F, Riancho-Zarrabeitia L, Díaz de Terán T, Miranda-Filloy JA, Blanco R, et al. Sex differences in patients with systemic lupus erythematosus from Northwest Spain. Rheumatol Int. 2014;34:11-24.

32. Brinks R, Hoyer A, Weber S, Fischer-Betz R, Sander O, Richter JG, et al. Age-specific and sex-specific incidence of systemic lupus erythematosus: an estimate from cross-sectional claims data of 2.3 million people in the German statutory health insurance 2002. Lupus Sci Med. 2016;3:e000181.

33. Arnaud L, Fagot J-P, Mathian A, Paita M, Fagot-Campagna A, Amoura Z. Prevalence and incidence of systemic lupus erythematosus in France: A 2010 nation-wide population-based study. Autoimmun Rev. 2014;13:1082-9.

34. Stefanidou S, Benos A, Galanopoulou V, Chatziyannis I, Kanakoudi F, Aslanidis S, et al. Clinical expression and morbidity of systemic lupus erythematosus during a post-diagnostic 5-year followup: a male:female comparison. Lupus. 2011;20:1090-4.

35. Boodhoo KD, Liu S, Zuo X. Impact of sex disparities on the clinical manifestations in patients with systemic lupus erythematosus: A systematic review and meta-analysis. Medicine (Baltimore). 2016;95:e4272.

36. Lu L-J, Wallace DJ, Ishimori ML, Scofield RH, Weisman MH. Review: Male systemic lupus erythematosus: a review of sex disparities in this disease. Lupus. 2010;19:119-29.

37. Faezi ST, Almodarresi MH, Akbarian M, Gharibdoost F, Akhlaghi M, Jamshidi A, et al. Clinical and immunological pattern of systemic lupus erythematosus in men in a cohort of 2355 patients. Int $\mathrm{J}$ Rheum Dis. 2014;17:394-9. 


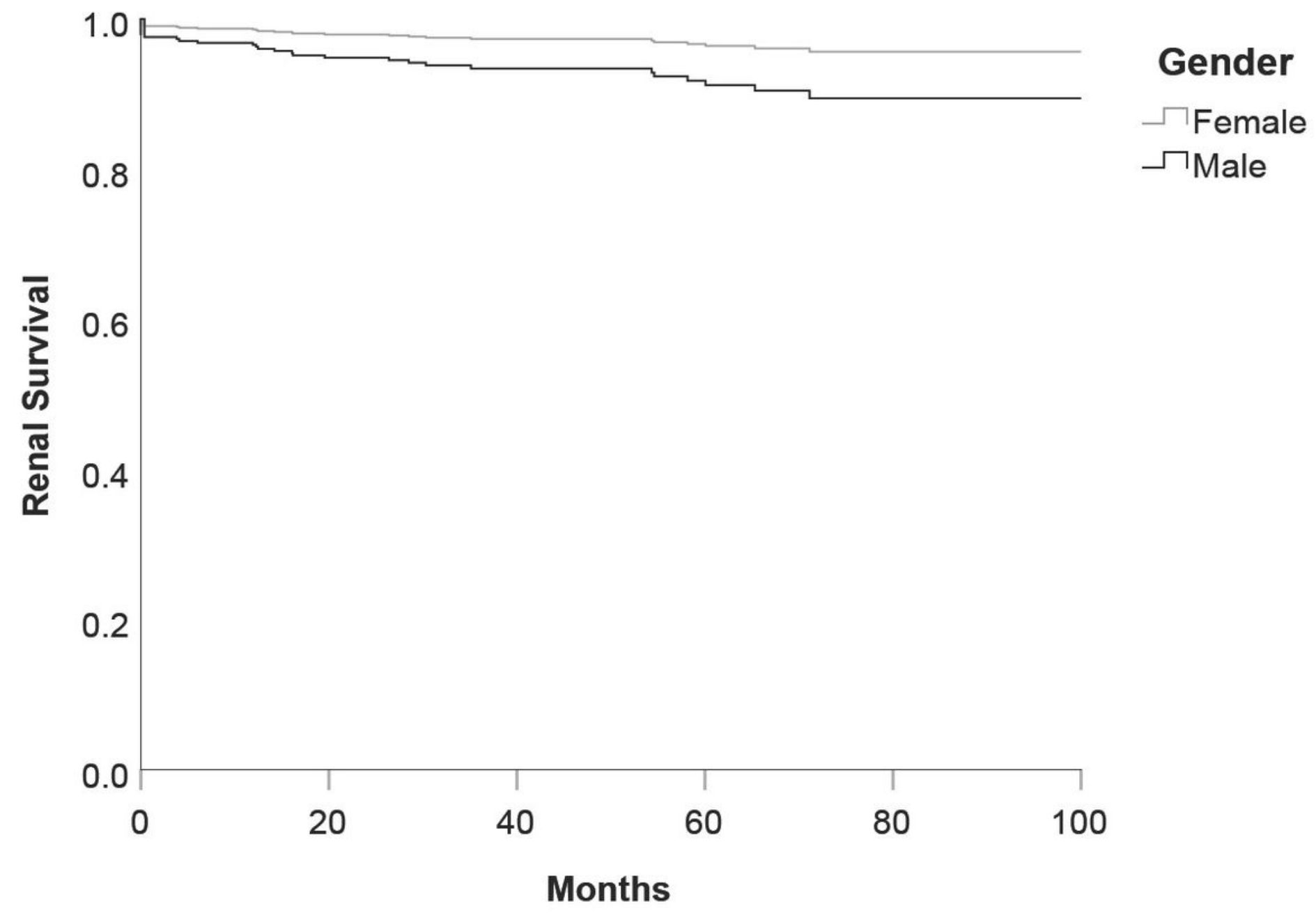

\section{Figure 1}

Cox regression survival curve for renal failure in men and women. Renal failure was defined as eGFR $<15$ $\mathrm{ml} / \mathrm{min} / 1.73 \mathrm{~m} 2$, initiation of renal replacement therapy, documented end stage renal disease in SLICCSDI or doubling of serum creatinine. Regression model included gender, age at inclusion, disease duration, time to diagnosis from disease manifestation, ethnicity, medication used during follow up, eGFR and SELENA SLEDAl at inclusion. 


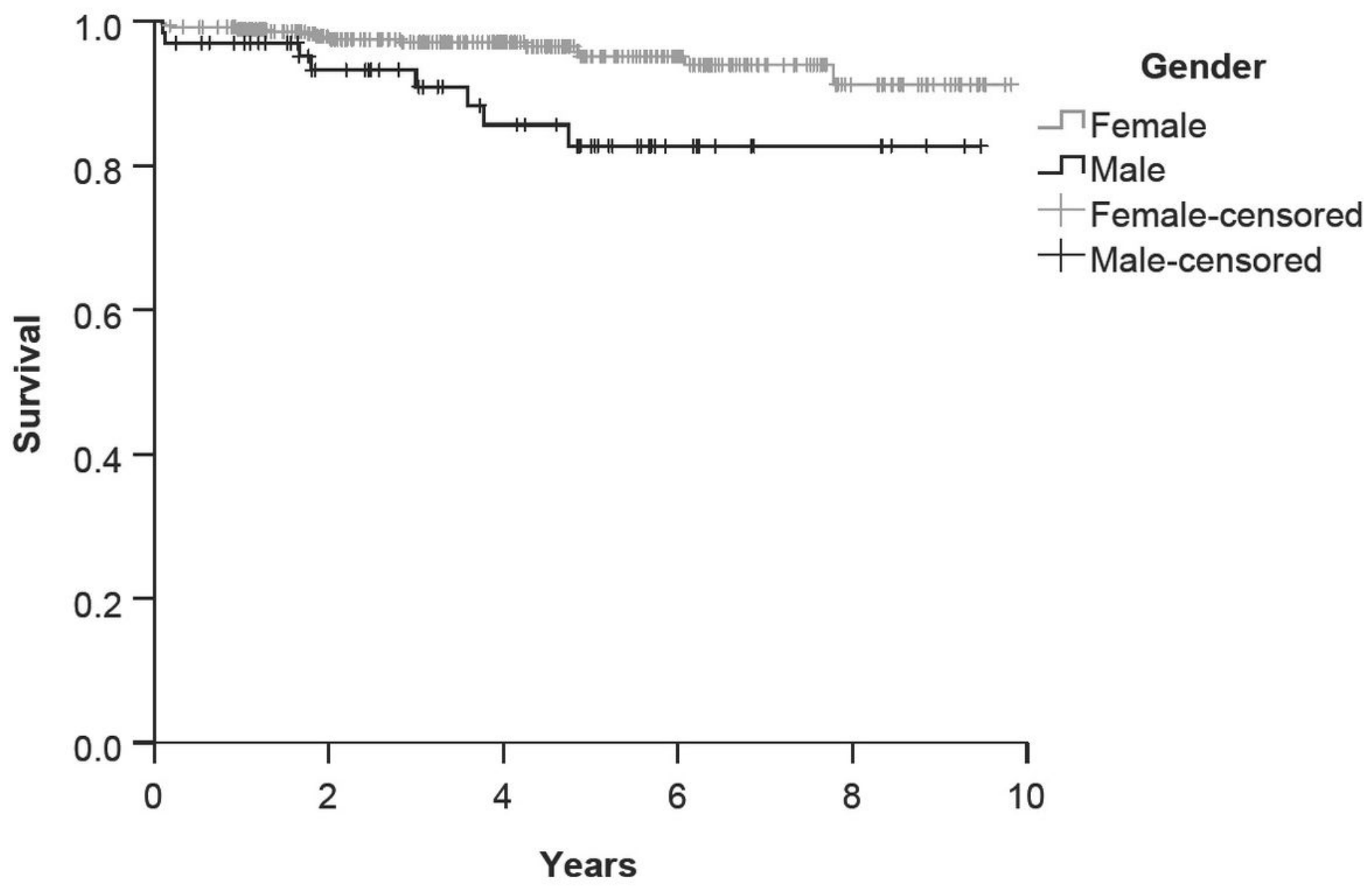

Figure 2

Kaplan Meier curve for overall mortality of male and female patients among study population.

\section{Supplementary Files}

This is a list of supplementary files associated with this preprint. Click to download.

- Supplementaryfile1.docx

- Supplementaryfile2.docx

- Supplementaryfile3.docx 